\title{
An Experimental Bacteriostasis of the Banxiaxiexin Decoction and 7 Kinds of Single Taste Traditional Chinese Medicine on Helicobacter Pylori Resistant Strains in Vitro
}

\author{
Zhiwei Qu ${ }^{1, \text { * }, \text { Mingjun } \mathbf{Y u}^{2} \text {, Airong Zhang }}{ }^{2}$, Ailin Wang ${ }^{3}$, Liyuan Shun ${ }^{3}$ \\ ${ }^{1}$ Department of Traditional Chinese Medicine, The Affiliated Hospital of Beihua University, Jilin, China \\ ${ }^{2}$ Department of Gastroenterology, The Affiliated Hospital of Beihua University, Jilin, China \\ ${ }^{3}$ Department of Laboratory Medicine, Beihua University, Jilin, China
}

Email address:

zhiweiqu@sina.com.cn (Zhiwei Qu)

${ }^{*}$ Corresponding author

\section{To cite this article:}

Zhiwei Qu, Mingjun Yu, Airong Zhang, Ailin Wang, Liyuan Shun. An Experimental Bacteriostasis of the Banxiaxiexin Decoction and 7 Kinds of Single Taste Traditional Chinese Medicine on Helicobacter Pylori Resistant Strains in Vitro. American Journal of Internal Medicine. Vol. 8, No. 3, 2020, pp. 138-142. doi: 10.11648/j.ajim.20200803.18

Received: April 19, 2020; Accepted: May 3, 2020; Published: May 28, 2020

\begin{abstract}
Objectives: To research experimental bacteriostasis on the Banxiaxiexin decoction and 7 kinds of single taste Traditional Chinese Medicine (TCM) for Helicobacter pylori resistant strains in vitro. Methods: Collecting specimens of gastric mucosa in patients with upper gastrointestinal disease, separated and identified 8 strains of Hp resistant strains, the E-test methods of metronidazole and clarithromycin, drug resistance status of amoxicillin, selected eight strains of clinical drug resistance strains; Using liquid dilution method to determine the Banxiaxiexin decoction and 7 kinds of single Chinese medicine clinical drug-resistant strains of 8 strains of antibacterial effect in vitro. Results: Banxiaxiexin decoction $\mathrm{MIC}$ is $0.31 \mathrm{mg} / \mathrm{ml}$, MIC of Radix scutellariae is $0.09 \mathrm{mg} / \mathrm{ml}$, Rhizoma coptidis MIC $0.16 \mathrm{mg} / \mathrm{ml}$, Glycyrrhizin realness MIC $0.31 \mathrm{mg} / \mathrm{ml}$, Ginseng MIC $0.75 \mathrm{mg} / \mathrm{ml}$, there are four of seven kinds of single taste Traditional Chinese Medicine of TCM has bacteriostatic action, the order of antibacterial strength: Scutellaria baicalensis $>$ Coptis chinesis $>$ Glycyrrhizin realness $>$ Ginseng, three kinds of Pinellia, Jujube and dry Zingibe in vitro for H. P ylori no inhibition, the method of MIC with liquid dilution in this decoction were determined, the decoction of resistant isolates average MIC isolates was $0.20 \pm 0.09 \mathrm{mg} / \mathrm{ml}$, the results showed that this decoction have stronger antibacterial effect on eight kinds of drug resistant strains in vitro. Banxiaxiexin decoction and 4kinds of single taste TCM have stronger antibacterial effect on drug resistant strains in vitro. Conclusion: Banxiaxiexin decoction and 4 kinds of single TCM have strong bacteriostatic effect on the bacteria resistance of Helicobacter pylori strains in vitro, therefore, we suggest that this decoction could be used as an effective method in the treatment of resistant HP associated gastritis.
\end{abstract}

Keywords: Helicobacter Pylori, Drug Resistance, Banxiaxiexin Decoction, Bacteriostatic Effect

\section{Introduction}

Helicobacter pylori is the cause of chronic active gastritis and duodenal ulcer. It is given priority to antibiotic combination scheme of $\mathrm{Hp}$ infection with effective treatment of western medicine [1], but along with the increase in antibiotic use, side effects and the resistant strains are also more and more [2-3]. From the perspective of Traditional Chinese Medicine to study the prevention and control drug resistant strains has opened up a new way, Banxiaxiexin decoction from zhang zhongjing in Chinese han dynasty [4],
Shang-han lun of prescriptions for Pinellia, Scutellaria baicalensis, Zingiber, Ginseng, Glycyrrhiza uralensis, Coptis chinesis, Jujube, the function may be modulated the cold and heat balance, eliminated swelling of the liver disease and dissipated nodules, Indicating on cold and heat mixed in the ruffian Syndrome as well as abdomen with swelling of the liver disease, but abdominal fullness without pain, or vomiting, bowel diarrhea, and yellowish greasy tongue. Clinical commonly we have used in Weak qi, cold and heat mutual knot, such as early acute or chronic gastroenteritis, chronic colitis, chronic hepatitis, cirrhosis of the liver [5-7]. We have 
curative effects on treating Helicobacter pylori related gastric disease in clinical observation of the decoction, especially resistant Helicobacter pylori cases, but the specific mechanism is unclear, therefore, we have researched experimental bacteriostasis on the decoction and 7 kinds of single taste Traditional Chinese Medicine (TCM) for Helicobacter pylori resistant strains in vitro, the purpose is to explore the decoction and 7 kinds of single Chinese medicine resistance mechanism of $\mathrm{H}$. pylori resistant strains, could be provided the objective basis for the prevention and treatment of H. pylori resistant strains.

\section{Methods}

\subsection{Patients and Sample Preparation}

A total of 105 patients seen at the Department of Gastroenterology, Affiliated Hospital of Beihua University, China, from July 2016 to August 2019 were included. The following cases were excluded: gastrointestinal cancer, surgical history, pregnancy and lactation, and so those with severe disease. Before gastroscopy, all the patients were confirmed that had not treated by any Hp eradication therapy (antibiotics, acid-suppressing drugs, NSAIDs or bismuth) within one month. The antibacterial test was performed with disc agar diffusion (K-B method). Drug resistant strains I VII were isolated and cultured from 105 patients' gastric mucosa, resistant to metronidazole, amoxicillin, clarithromycin, ornidazole, levofloxacin, moxifloxacin and rifampin, as well as Susceptibility testing to etronidazole amoxicillin, clarithromycin, ornidazole, levofloxacin, moxifloxacin and rifabutin was performed with disc agar diffusion method (Bio Merieux, France), results determine reference [8-9]. Strains were classified as resistant when the MIC (minimal inhibitory concentration) was $\geq 8 \mu \mathrm{g} / \mathrm{ml}$ (etronidazole); $\geq 0.5 \mu \mathrm{g} / \mathrm{ml}$ (amoxicillin); $\geq 1 \mu \mathrm{g} / \mathrm{ml}$ (clarithromycin); $\geq 2 \mu \mathrm{g} / \mathrm{ml}$ (ornidazole); $\geq 2 \mu \mathrm{g} / \mathrm{ml}$ (levofloxacin); $\geq 2 \mu \mathrm{g} / \mathrm{ml}$ (moxifloxacin); $\geq 2 \mu \mathrm{g} / \mathrm{ml}$ (rifampicin). The standard strain NCTC11637 was donated by Professor Hu Fulian of the First Affiliated Hospital of Peking University.

\subsection{Chinese Medicine Reagent}

We have selected the Chinese medicinal materials of banxiaxiexin decoction as follows: Put Pinellia (9 g), Scutellaria baicalensis (6 g), Zingiber (6 g), Ginseng (6 g), Glycyrrhiza uralensis (6 g), Coptis chinensis (3 g), Jujube (3 g) in $500 \mathrm{ml}$ water, simmered for $2 \mathrm{~h}$, filtered residue and added water to continue boiling for $30 \mathrm{~min}$, filtration to get the final concentration (each $100 \mathrm{ml}$ liquid contained $10 \mathrm{~g}$ of original drugs), Chinese medicine, Pinellia, Radix scutellariae, Ginseng, Rhizoma coptidis, Radix glycyrrhizae, Jujube, Dried ginger, were purchased from Sanjiu Pharmaceutical Co., Ltd, China.

\subsection{Isolation and Culture of H. Pylori}

Specimen collection and HP separation and culture: through gastroscope, a piece of antrum and body of stomach were taken respectively with biopsy forceps within $5 \mathrm{~cm}$ from the front of pylorus, used sterile forceps to put the specimen into a centrifuge tube filled with delivery solution, placed it in $4^{\circ} \mathrm{C}$ for preservation and transferred to the laboratory. In the super clean table of aseptic room, a piece of gastric mucosa tissue were taken, pressed the mucosa tissue with inoculation ring and smeared it on skirrow culture medium (Shanghai Yubo Biological Technology Co., Ltd, China); poured skirrow culture medium into anaerobic tank for 3 days, HP culture conditions: $37^{\circ} \mathrm{C}, 10 \% \mathrm{CO} 2,5 \% \mathrm{O} 2,85 \% \mathrm{~N} 2$. After $3-5$ days of culture, the transparent needle like colonies on the agar plate were selected, transferred to a new skirrow selective medium, and the strains were purified and enriched.

\subsection{Identification of HP Bacteria}

Morphological examination: the HP colonies were inoculated by scribing line showed transparent needle tip shape, neat edge, central protuberance and diameter of 1.2 ram colony; a translucent layer of lichen when inoculated with large amount of bacteria. Select the typical HP colony, smeared it in a drop of normal saline, dry it, and then gram stain it, and observed it under ordinary microscope. Seeing gram-negative S-shaped Campylobacter or Brevibacterium (see Figure 1). It can be preliminarily determined as HP. 2) Biochemical examination: taking HP colony for urease, catalase and oxidase test (Nanjing dulai Biotechnology Co., Ltd, China). If all three biochemical reactions are positive, HP were determined as:

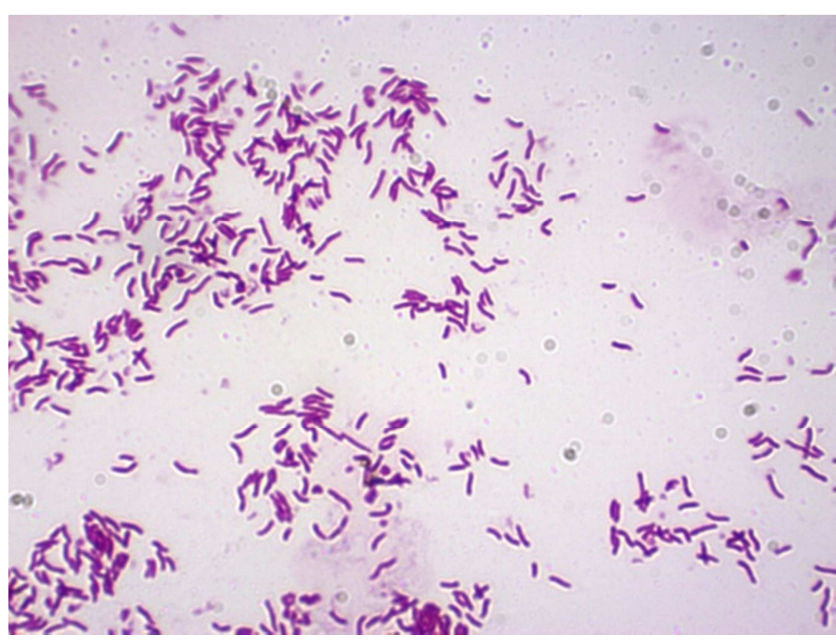

Figure 1. Morphology of Helicobacter pylori $10 \times 100$.

HP preservation: We have selected the third generation HP of enrichment culture, and preserved it in its logarithmic growth period (General 48 hours); added $3-5 \mathrm{ml}$ of preservation solution ( $30 \%$ glycerol bouillon) to elute the bacteria on the agar surface, used aseptic straw to separate the bacterial solution into $1.5 \mathrm{ml}$ aseptic eppend of tube or cryopreservation tube, and quickly placed the strain in $-80^{\circ} \mathrm{C}$ refrigerator for preservation.

HP recovery: the bacteria were taken out directly from the refrigerator, unfreezed at room temperature, used the sterile 
elbow pipette to absorb the bacteria solution, dropped it on the skirrow selective culture medium, used the L-shaped glass rod to evenly coat the bacteria solution, and putted the culture dish into the incubator for incubation. the conditions were the same as before. Drug sensitivity test: we have collected fresh HP from pure culture for 48-72 hours and fully grind it into sterile saline, adjust the concentration to $2 \times 108 \mathrm{cfu} / \mathrm{ml}$, dipped the sterile cotton swab in the bacterial solution, evenly coated the whole agar surface, stand, slightly dry, took the E-test strip containing antibiotics and laid it flat on the agar surface (1 / 90 $\mathrm{mm}$ plate), $37^{\circ} \mathrm{C}$, and cultured in microaerobic environment for 72 hours, the results showed that the minimum inhibitory concentration (MIC) was the scale at the junction of the ellipsoidal bacteriostatic ring and the test strip.

\subsection{Determination of MIC}

MIC were determined by liquid multiple dilution method: experiment study on antimicrobial Banxiaxiexin decoction and seven kinds of single herbs resistant strains of $\mathrm{H}$. pylori in vitro assays were proformed. Concentration Banxiaxiexin decoction were $10 \mathrm{mg} / \mathrm{ml}$, the concentration of each herb was: Pinellia $15 \mathrm{mg} / \mathrm{ml}$, Scutellaria baicalensis $6 \mathrm{mg} / \mathrm{ml}$, Zingiber $12 \mathrm{mg} / \mathrm{ml}$, Ginseng12 mg/ml, Glycyrrhiza uralensis $10 \mathrm{mg} / \mathrm{ml}$, Coptis chinensis $10 \mathrm{mg} / \mathrm{ml}$, Jujube $6 \mathrm{mg} / \mathrm{ml}$. Took 8 holes of 48 holes microtiter plate, added $1 \mathrm{ml}$ of liquid medium into each hole, and $200 \mu 1$ of the above preparation solution into the first hole, shaking well, and diluted to the eighth hole by multiple ratio. the drug concentration were $128,64,32,16,8$, 4, $2 \mathrm{mg} / \mathrm{ml}$ in turn. In each tube, $10 \mu 1$ of liquid culture HP bacterial suspension was added, and the eighth hole was negative control. Microaerobic environment, $120 \mathrm{R} / \mathrm{min}$, shaking culture $48 \mathrm{~h}$. The turbidity of the culture plate was observed with naked eyes, and the samples were stained and biochemical identified. At the same time, $100 \mu 1$ was taken to the solid medium for further culture for 72 hours, and the growth of the colony was observed. If there was no bacterial growth, it was judged that it had bacteriostatic effect, If it is found that bacteria grow on the medium containing the highest concentration of $0.31 \mathrm{mg} / \mathrm{ml}$, its MIC value is calculated as $0.31 \mathrm{mg} / \mathrm{ml}$.

\section{Results}

The results of Banxiaxiexin decoction and 7 kinds of single taste TCM for HP standard strains in vitro bacteriostasis experiment MIC are shown in table 1 the decoction and 7 kinds of single Chinese medicine MIC were determined by liquid dilution, the results showed that the Banxiaxiexin decoction and 7 kinds of single taste TCM for HP, strong bacteriostatic activity of Banxiaxiexin decoction $\mathrm{MIC}$ is 0.31 $\mathrm{mg} / \mathrm{ml}$, Radix scutellariae MIC $0.09 \mathrm{mg} / \mathrm{ml}$, Rhizoma coptidis MIC $0.16 \mathrm{mg} / \mathrm{ml}$, Licorice MIC $0.31 \mathrm{mg} / \mathrm{ml}$, Ginseng MIC 0. $75 \mathrm{mg} / \mathrm{ml}$, there are four kinds of Chinese medicine in $7 \mathrm{kinds}$ of single taste TCM, the strength of inhibitory effects followed as: the Scutellaria baicalensis, Coptis chinensis, Glycyrrhiza uralensis, Ginseng, However, the strongest one is Scutellaria and weakest Ginseng, but no inhibition in three kinds of Pinellia, Jujube and Dry zingibe in vitro.

Table 1. MIC value of Banxia Xiexin Decoction and 7 kinds of single Chinese herbs against H. pylori (mg/ml).

\begin{tabular}{llll}
\hline Chinese pinyin & English name & Latin name & MIC limits \\
\hline Banxia Xiexin Tang & Banxia Xiexin Decoction & Banxia Xiexin Decoction & $32 \sim 128$ \\
Huang qin & Baikal Skullcap & Scutellaria baicalensis & $64 \sim 128$ \\
Huang lian & Coptis chinensis & Coptis chinensis Franch & $64 \sim 128$ \\
Gan cao & Licorice & Glycyrrhiza uralensis & $32 \sim 128$ \\
Ren shen & Ginseng & Panax schinseng & $16 \sim 128$ \\
Banxia & Pinelliae Rhizoma & Pinellia ternatab & $2 \sim 128$ \\
Aan jiang & Dried ginger & Zingiber officinale Rosc & $2 \sim 128$ \\
Da zao & Jujube & Ziziphus jujuba Mill & 0.31 \\
\hline
\end{tabular}

Table 2. MIC of Banxia Xiexin Decoction to 8 drug resistant strains by continuous dilution of solid medium.

\begin{tabular}{lll}
\hline Bacterial strain & MIC limits & MIC50 \\
\hline Standard strain & $32 \sim 128$ & 0.31 \\
Isolate 1 & $32 \sim 128$ & 0.31 \\
Isolate 2 & $16 \sim 128$ & 0.15 \\
Isolate 3 & $8 \sim 128$ & 0.08 \\
Isolate 4 & $16 \sim 128$ & 0.15 \\
Isolate 5 & $32 \sim 128$ & 0.31 \\
Isolate 6 & $16 \sim 128$ & 0.15 \\
Isolate 7 & $32 \sim 128$ & 0.31 \\
Isolate8 & $16 \sim 128$ & 0.15 \\
\hline
\end{tabular}

This decoction to eight kinds of resistant strains of bacteriostatic effect in vitro bacteriostasis experiment MIC is shown in table 2, the MIC of Banxiaxiexin decoction were determined by liquid dilution method, the results showed that Banxiaxiexin decoction is stronger to 8 kinds of resistant strains in bacteriostatic activity, standard strains of MIC is $0.31 \mathrm{mg} / \mathrm{ml}$, Isolates $1 \mathrm{MIC} 0.31 \mathrm{mg} / \mathrm{ml}$, Isolates $2 \mathrm{MIC} 0.15$ $\mathrm{mg} / \mathrm{ml}$, Isolates $3 \mathrm{MIC} 0.08 / \mathrm{mg} / \mathrm{ml}$, Isolates 4 MIC 0.15 $\mathrm{mg} / \mathrm{ml}$, Isolates $5 \mathrm{MIC} 0.31 \mathrm{mg} / \mathrm{ml}$, Isolates $6 \mathrm{MIC} 0.15$ $\mathrm{mg} / \mathrm{ml}$, Isolates $7 \mathrm{MIC} 0.31 \mathrm{mg} / \mathrm{ml}$, Isolates $8 \mathrm{MIC} 0.15$ $\mathrm{mg} / \mathrm{ml}$, the average MIC were $0.20 \pm 0.09 \mathrm{mg} / \mathrm{ml}(\mathrm{x} \pm \mathrm{SD})$, So that the decoction have strong effects on antibacterial in vitro against 8 kinds of drug resistant strains.

\section{Discussion}

At present western medicine for Helicobacter pylori eradication therapy is most typically applied to clinical antibiotic therapy, Containing the PPI triple therapy is one of the most admired the preferred therapy (omeprazole + metronidazole, amoxicillin and clarithromycin in any [10-11]. Although there are a lot of western medicine antibiotic 
therapy clinical studies have shown that its eradication of Helicobacter pylori is very effective, but there are still many problems of therapy, including poor patient compliance, drug side effects, may be caused disorder in patients with gastrointestinal flora, and broke out Superinfection repeatedly, especially because of the rapid increase of drug-resistant strains in recent years, the clinical efficacy of antibiotic therapy has declined in varying degrees [12]. For these reasons, many researchers at home and abroad are gradually broadening their research ideas of eradicating $\mathrm{H}$. pylori and seeking effective drugs to treat drug-resistant $\mathrm{H}$. pylori. TCM in the eradication of $\mathrm{H}$. pylori advantages also gradually outstanding. TCM has a little side effects, the advantages of high safety, not easy the creation of drug resistant, therefore, the treatment of $\mathrm{Hp}$ infection will have broad prospects in the clinical practice, our study showed that seven kinds of four kinds of Chinese medicine has antibacterial, the results consistent with the literature [13, 14], the order of antibacterial strength is Scutellaria, Coptidis, Glycyrrhizae, Ginseng, but, Pinellia, Jujube and Zingiber have no significant inhibition in vitro.

Chinese medicine theory research has showed that $\mathrm{H}$. pylori is a kind of "evil" invading the body [15]. Concrete said that $\mathrm{H}$. pylori is a "wet and heat toxin factor", wetlands for yin evil influence, easily injured the spirit of yang qi and the liquid viscosity, hindered the qi activity, therefore, It is difficult to eradication of $\mathrm{H}$. pylori, the evils violations, diet could damage the stomach, feeling unhappy and unobstructed also may increase the chance of $\mathrm{H}$. pylori infection caused by an important cause in gastrointestinal disease, and the Banxiaxiexin decoction with detoxification, health spleen and reduce moisture, the system adjustable gastric acid function and analgesic efficacy of mitigation [16], Single pharmacological study of Coptis and Scutellaria in the formula has the effect on killing HP bacteria; Pinellia may be reconciled the stomach and resolved moisture, encouraging stomach, so enhanced the body's resistance to diseases, protected gastric mucosa, promoting repair of tissue damage [17-19]; Glycyrrhizae have effects on inhibition of gastric acid, changed the stomach of PH easily, destroyed the HP survival environment, accelerated the HP death [20-21]; Ginseng flavored sweet has the functions of tonifying and improving immunity, as well as could increase IL 6 and IL 8 in blood [22]; Jujube is sweet and warm, running benefit the spleen and stomach fluids, various drugs were played at reconciling health spleen stomach, defusing the effect of heat and moisture, our results show that this decoction for resistant $H$. pylori strains have stronger antibacterial activity in vitro. may be prompted the future of the TCM to develop a new generation of HP drug resistance, contribution to the eradication of HP infection treatment, the mechanism of antibacterial activity needs us to continuous research in the future.

\section{Abbreviations}

$\mathrm{HP}=$ Helicobacter $\quad$ pylori, $\quad \mathrm{TCM}=$ Traditional Chinese Medicine, $\mathrm{MIC}=$ Minimum inhibitory concentration.

\section{Acknowledgements}

Professor Mingcheng Li from College of Medical Laboratory Science of Beihua University have contributed to the revision and finalization of later manuscripts, and Professor Haiying Gu from department of microbiology of Hainan medical college have given great help to microbiological test.

\section{References}

[1] Julia Silveira, Ivy Bastos, Daniela Fernandes, Drug resistance in Helicobacter pylori 1. Arq Gastroenterol. 2016, 53 (4): 215-223.

[2] Takeuchi H, Morimoto N, Sugiura T. Molecular-pathological analysis of Helicobacter pylori-associated disorders and clinical laboratory testing. Rinsho Byori. 2014; 62 (5): 440-9.

[3] Bachir M, Allem R, Tifrit A, et al. Primary antibiotic resistance and its relationship with cagA and vacA genes in Helicobacter pylori isolates from Algerian patients. Braz J Microbiol. 2018, 2 (13): S1517-8382.

[4] Li Peisheng, lecture of Shang-han lun Shanghai Science and Technology Press, first edition, 1985, 88.

[5] Zhu Cuiling, Shiqiao, Zhou Lijuan, etc., Study on the treatment of spleen and stomach disease with Banxia Xiexin Decoction, Journal of Changchun University of Traditional Chinese Medicine2013, 29: 347-349.

[6] Jiaxing Tian, Min Li, Jiangquan Liao, Chinese Herbal Medicine Banxiaxiexin Decoction Treating Diabetic Gastroparesis: A Systematic Review of Randomized Controlled Trials. Evidence-Based Complementary and Alternative Medicine 2013, 7 (11): 1-6.

[7] GanY, LiuH, YangL, YangK. Effect of banxiaxiexin tang on treatment of functional dyspepsia: a meta-analysis of randomized controlled trials. J Tradit Chin Med. 2014; 34 (2): 140-144.

[8] Boyanova L, Gergova G, Nikolov R, Derejian S, Lazarova E, Katsarov $\mathrm{N}$ et. al, Activity of Bulgarian propolis against 94 Helicobacter pylori strains in vitro by agar-well diffusion, agar dilution and disc diffusion methods. J Med Microbiol. 2005; 54 (5): 481-3.

[9] McNulty C, Owen R, Tompkins D, Hawtin P, McColl K, Price A et. al, Helicobacter pylori susceptibility testing by disc diffusion. J Antimicrob Chemother. 2002; 49 (4): 601-9.

[10] Tajana Filipec Kanizaj, Nino Kunac Helicobacter pylori: Future perspectives in therapy reflecting three decades of experience, World J Gastroenterol 2014, 21; 20 (3): 699-705.

[11] Novosti Buta1, Nicoline F. Tanih1 and Roland N. Ndip, Increasing trend of metronidazole resistance in the treatment of Helicobacter pylori infection: A global challenge, African Journal of Biotechnology, 2010. 9 (8): 1115-1121.

[12] Ducournau A, Bénéjat L, Sifré E, Bessède E et al. Helicobacter pylori resistance to antibiotics in 2014 in France detected by phenotypic and genotypic methods. Clin Microbiol Infect. 2016, 22 (8): 715-8. 
[13] Chen Meihong, Yan Jin, Dang Yini et al., Advances in Study on Antibiotic Resistance of Helicobacter pylori, Chin J Gastroenterol 2019, 24 (2): 511 -118.

[14] Feng Ma, Ye Chen, Jing Li, He-Ping Qing, et al. Screening test for anti- Helicobacter pylori activity of traditional Chinese herbal medicines, World J Gastroenterol 2010; 16 (44): $5629-5634$

[15] Jiang Lin, Wei-Wen Huang, A systematic review of treating Helicobacter pylori infection with Traditional Chinese Medicine, World J Gastroenterol 2009; 15 (37): 4715-4719.

[16] Kang B, Lee H, Choi Y, Jeon C, Lee JA. Banxia Xiexin tang for gastro-oesophageal reflux disease, A protocol for a systematic review of controlled trials, Medicine (Baltimore). 2018; 97 (17): e0393.

[17] Mengjiao Hu1, Zhenfei Wang, Treatment of Helicobacter pylori-associated gastritis using traditional Chinese medicine, Infection International; 2018; 7 (1): 1-5.

[18] Ye Hui, Shi Zong-ming, Chen Yao, Yu Jing, Innovative
Perspectives of Integrated Chinese Medicine on H. pylori, Chin J Integr Med, 2018; 24 (11): 873-880.

[19] Yao Dongting, Hu Jun, Zhang Xueqing, Hu Xiaobo, Inhibitory activity of Chinese medicine coptis chinensisfranch against Staphylococcus aureus in vitro. laboratory medicine 2017, 32 (7): 577-581.

[20] Zhang Tao, Zhang Xiaohu, Shen Yanxia, Yin Jiaqi, preliminary study on its antibacterial effects in baicalein from extraction of Scutellaria baicalensis. Journal of Shaanxi Agricultural Science, 2018, 64 (04): 53-57.

[21] Chai Fang, Li Junyu, Zhang Haifeng, Zhou Xiangfu, Zhu Xiong, Chen Hai, Skullcap Capsule on Bacteriostasis in vitro of 193 Strains of Multi - Drug Resistant Acinetobacter baumannii. Chinese Archives of Traditional Chinese Medicine, 2016; 34 (11): 2694-2696.

[22] Lee JS1, Hwang HS2, Ko EJ et. al Immunomodulatory activity of red ginseng against influenza A virus infection, Nutrients. 2014; 6 (2). 